\title{
Guest Editorial \\ Microassembly for Manufacturing \\ at Small Scales
}

$\mathbf{M}$

ICROELECTRONICS brought an information revolution through integrating a vast number of microscopic transistors. Much progress has been made in miniaturization and integration of MEMS or MOEMS (Micro-(Opto-)Electro-Mechanical-Systems to produce accelerometers, inkjet printer heads, micro-mirrors, micro-relays, and pressure sensors. A new generation of MEMS is rapidly moving toward highly integrated, more complex heterogeneous microsystems with increased functionalities. Many limitations remain that are extremely difficult to overcome, especially concerning processes and materials incompatibilities. microassembly is a natural and powerful approach to overcome these processes incompatibilities and to facilitate complex, heterogeneous, $3 \mathrm{D}$, or out of plane integration. By using basic micro-scale components, microassembly constitutes a new alternative of microsystems production that may lead to cost savings and shorter development cycle times. Because of the size of the components and of the required precision, automation is needed.

Recent developments enabled proofs of concept (actuators, sensors, gripping principles and tools, control, manipulation, etc.) but assemblies performed automatically and/or with acceptable yields are extremely challenging. It requires simultaneously consider microfabrication tolerances $(\mathrm{M}(\mathrm{O})$ EMS components to assemble, handling tools, etc.), robot uncertainties, sensor integration, reliable control, bonding and metrology aspects, all of these aspects being specific at the microscale (lack of sensors and adapted gripping tools, small signal to noise ratio, use of nonlinear actuators, surface force predominance, etc.).

This Special Section presents recent and original advances in microassembly methods and technologies.

This Special Section opens with a survey paper on "Research in Automated Planning and Control for Micromanipulation," by Banerjee and Gupta. A level of automation is necessary as it is challenging to manually control the microobjects due to the scaling effect of the surface forces, stochastic motion of objects in fluid media, and uncertainty associated with object state estimation.

The second survey paper is entitled, "A Review for Haptic Feedback Teleoperation Systems for Micromanipulation and Microassembly," by Bolopion and Régnier. Haptic feedback teleoperation, where the user manipulates the tool through a joystick while feeling a force feedback, is a promising solution as it allows high intuitiveness and flexibility for micromanipulation and microassembly.

The paper "Precision Position/Force Interaction Control for a Piezoelectric Multimorph Microgripper for Microassembly" by Xu presents two new approaches to simultaneously regulate both position and contact force of a piezoelectric multimorph microgripper dedicated to microassembly tasks.

The paper "Automated Guiding Task of a Flexible Micropart Using a Two-Sensing-Fingers Microgripper" by Komati, Rabenorosoa, Clévy, and Lutz, presents a control strategy using an external hybrid force/position control and taking into account microscale specificities. It is very accurate, with high speed (low rejection time) and easy to implement. It is noted that the proposed control scheme can also be applied to other microassembly tasks (pick-and-place, insertion, etc.).

The paper by Pac and Popa, entitled "Interval Analysis of Kinematics Errors in Serial Manipulators Using Product of Exponentials Formula" deals prediction of end-effect or pose deviation from its ideal model using the interval bounds on the errors of the individual axes. Intervals offer a mathematically concise and computationally efficient tool for analysis and design of precision robots.

The paper "Magnetic Self-Assembly of Ultra-Thin Chips to Polymer Foils" by Tichem proposes a self-assembly process for the placement and alignment of ultra-thin chips (UTCs) to polymer foils.

In the paper, "Analysis and Motion Control of a CentrifugalForce Microrobotic Platform," by Panaqiotis Vartholomeos, Vlachos, and Papadopoulos, the low cost, rapid fabrication, and micrometer motion resolution suggest that this microrobotic platform is a promising solution for low cost microfactories, where a group of such robots performs high throughput, advanced microassembly of microsystems.

The last paper of the Special Section is entitled, "Automated Pick and Place of Silicon Nanowires," by Ye, Zhang, Ru, Luo, Xie, and Sun. Nanowire pick-and-place has been typically performed via teleoperation, which is time-consuming and skill-dependent. This paper presents an automated approach

The guest editors would like to thank the members of the T-ASE Editorial Board, in particular, Prof. K. Goldberg, 
Editor-in-Chief, for the opportunity to publish this Special Section, and the Editorial Assistants, F. Agnew and J. Barbato, for their assistance. We would like to give a special thanks to the authors of the papers and to the reviewers.

PHILIPPE LUTZ, Guest Editor

FEMTO-ST, UMR CNRS 6174-UFC/ENSMM/UTBM

University of Franche-Comté

Besancon, 25000 France

philippe.lutz@femto-st.fr

KARL F. BÖHRINGER, Guest Editor

Department of Electrical Engineering and Bioengineering

University of Washington

Seattle, WA 98195-2500 USA

karlb@washington.edu
CÉDRIC ClÉvy, Guest Editor

FEMTO-ST, UMR CNRS

6174-UFC/ENSMM/UTBM

Department of Automatic Control and

Micro-Mechatronic Systems (AS2M)

Besancon, 25000 France

cedric.clevy@femto-st.fr

DAN POPA, Guest Editor

University of Texas at Arlington

Arlington, TX 76019 USA

popa@uta.edu

QUAN ZHOU, Guest Editor

Department of Automation and Systems Technology

School of Electrical Engineering

Aalto University

Aalto, 00076 Finland

quan.zhou@aalto.fi 